\title{
Optimization of 3D Printing Process
}

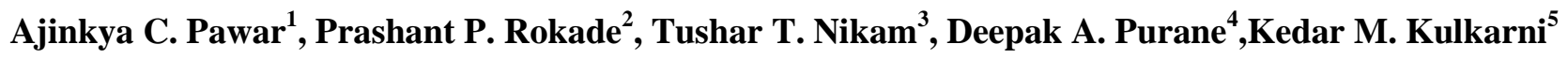 \\ Student, Department of Mechanical Engineering, KJEI's Trinity Academy of Engineering, Pune ${ }^{1,2,3,4}$ \\ Assistant Professor, Department of Mechanical Engineering, KJEI's Trinity Academy of Engineering, Pune ${ }^{5}$
}

\begin{abstract}
Technology of 3D-Printer based on fused deposition material has developed intensively with varying qualities. However, default setting of printing process parameters provided by the manufacturer in some cases does not guarantee quality (dimension error and strength) of the printed part, since there are several process parameters that need to be considered. A 3DPrinter with poly-lactic acid filament material has been applied in this study. A specimen standard has been used as a tensile strength and dimension error test to represent printed part quality. Three printing process parameters: layer thickness, layer speed and infill density have been optimized using Taguchi L9 Orthogonal Array method. In a recent project, we have thus investigated possibilities to increase the printability of especially flexible filament. The presentation will give an overview of the modifications and their effect on the most frequently used elastic filaments, offering suggestions to modify common 3D FDM printers in similar ways for increased printing results with or without integrated fibres or filaments.
\end{abstract}

Keywords: Optimization 3D Printing Process, Fused Deposition Modelling, L9 Orthogonal Array Method, Input Parameters

\section{INTRODUCTION}

Optimization of process parameters is done to have great control over quality, productivity and cost aspects of the process. Fused Deposition Modelling (FDM) is an Additive Manufacturing Technology for printing 3D objects layer by layer. Rapid Prototyping (RP) is the term given to a set of processes that can quickly fabricate any given threedimensional object into a model or prototype, directly from a CAD file via the additive deposition of individual crosssectional layers of the part.

\section{LITERATURE REVIEW}

In this chapter the literature review about "Optimization of 3D printing process" is done. Some views of scientist are discussed in this chapter

$>$ Panda et. al. concluded that if number of layers is more, it will result in high temperature gradient towards the bottom of part. This will increase diffusion between adjacent raster and strength will improve

$>$ Farzad et. al. said that the models derived serve as predictive models which can be used to anticipate the theoretical best parameter setting that would result in optimal response characteristics.

$>$ B. S. Raju et. al. said that optimization is done by Taguchi technique to know the effect of parameter on the variables which can be determined by integrating the Taguchi method with Grey Relational Analysis.

$>$ Shyam Kumar Karna and Dr. Rajeshwar Sahai has appraised on "Taguchi started to develop new methods to optimize the process of engineering experimentation. He believed that the best way to improve quality was to design and build it into the product. He developed the techniques which are now known as Taguchi Methods. His main contribution lies not in the mathematical formulation of the design of experiments, but rather in the accompanying philosophy".

> C K Basavaraj and M Vishwas has revised on "Process parameters were optimized for FDM process using Taguchi's L9 orthogonal array. Significant process parameters were identified using ANOVA. The set of process parameters which give optimum results. The percentage contribution shows the effect of process parameter on response characteristics. Because the thinner layer thickness gives better bonding strength and gives good axial loading capability. When the orientation angle changes the bonding strength between layers is varied with layer thickness."

$>$ Srinivas Athreya and Dr Y.D.Venkatesh has studied on "the paper illustrates the application of the parameter design (Taguchi method) in the optimization of facing operation. The following conclusions can be drawn based on the above experimental results of this study: Taguchi's Method of parameter design can be performed with lesser number of experimentations as compared to that of full factorial analysis and yields similar results. Taguchi's method can be applied for analyzing any other kind of problems as described in this paper. It is found that the 


\title{
International Advanced Research Journal in Science, Engineering and Technology
}

\author{
Vol. 6, Issue 3, March 2019
}

parameter design of the Taguchi method provides a simple, systematic, and efficient methodology for optimizing the process parameters."

$>$ Vinod G. Surange and Punit V. Gharat has appraised on "this paper to investigate the effects of raster angle and layer thickness on mechanical properties of 3Dprinted samples. The experiments confirmed that raster angle and layer thickness both have a marked effect on tensile, compressive and three-point.In this study, the mechanical properties of 3D-printed PEEK samples (tensile, compressive and three-point bending) were higher than those of ABS samples printed by commercial 3D printers. Specifically, the tensile, compression and bending strengths of PEEK samples were higher than those of ABS samples."

$>$ Prof. Deepa yagnik has revised on "The intention behind the research is to develop a low cost 3D Printer by using materials which are easily available and cost effective. We have been successful in reducing the cost to a considerable extent i.e about 10-15\%. The parts made in 3D design software are successfully imported in the printing software and the product obtained has the same dimension given during the design stage of the product i.e an accuracy close to $100 \%$.We were able to successfully fabricate the $3 \mathrm{D}$ printer according to its virtual design proposed at reduced cost".

\section{METHODOLOGY}

In this chapter the methodology about "Optimization of 3D printing process" is done. Some steps are discussed.

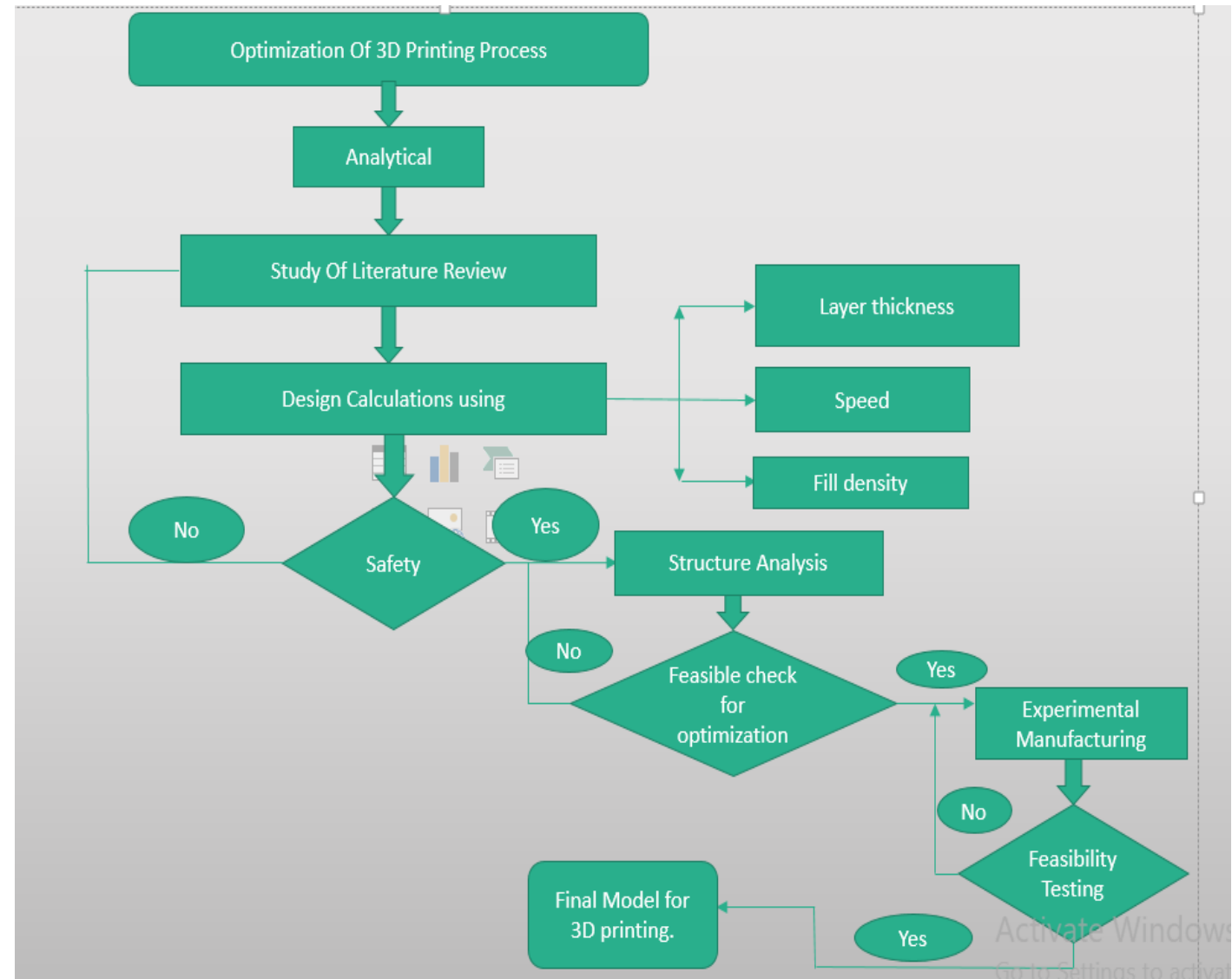

Figure. 1. Methodology of Proposed Optimization Process

\section{WORKING PRINCIPLE AND MODEL DESIGN}

The working principle on optimization process with catia model is discussed below.

\subsection{Working Principle}

$>$ The working of FDM machine generally consists of 3 steps: Pre-processing, Production and Post-production.

$>$ Pre-processing:- The pre-processing represents the very first steps in any additive manufacturing. This includes readying the CAD file for "printing," or building. The proprietary software performs as described above, creating a code that translates into mechanical movements and thermal adjustments performed by the FDM machines. Build 


\title{
International Advanced Research Journal in Science, Engineering and Technology
}

\author{
Vol. 6, Issue 3, March 2019
}

preparation software slices and positions a 3D CAD file and calculates a path to extrude thermoplastic and any necessary support material.

$>$ Production:- The 3D printer heats the thermoplastic to a semi-liquid state and deposits it in ultrafine beads along the extrusion path. Where support or buffering is needed, the $3 \mathrm{D}$ printer deposits a removable material that acts as scaffolding. The extrusion head heats up to the appropriate temperature. When thermally stable, the head pulls the build material and support material from their perspective storage spools. These thermoplastic filaments melt at the extrusion head and deposit as a bead being laid onto the build platform. Simultaneously, the extrusion head moves along the tool paths that were pre-programmed for each layer. As the tool path of that specific layer is completed, the build platform descends vertically in a very small incriminate. This vertically aligns the extrusion head with the next layer. The thermoplastics solidify almost instantly upon entering the temperature-controlled build chamber, which builds the part layer by layer, from bottom to top. Support materials are built under overhanging sections of the part.

$>$ Post-processing:- As the extrusion head finishes depositing material, the build chamber and build platform remain mechanically and thermally stable. This allows the build part and support structures to settle and cure with proper microstructures. After this cooling stage, the support structures are broken away and the final build part is cleaned. Thus, the fused deposition modelling process has come to a finish.

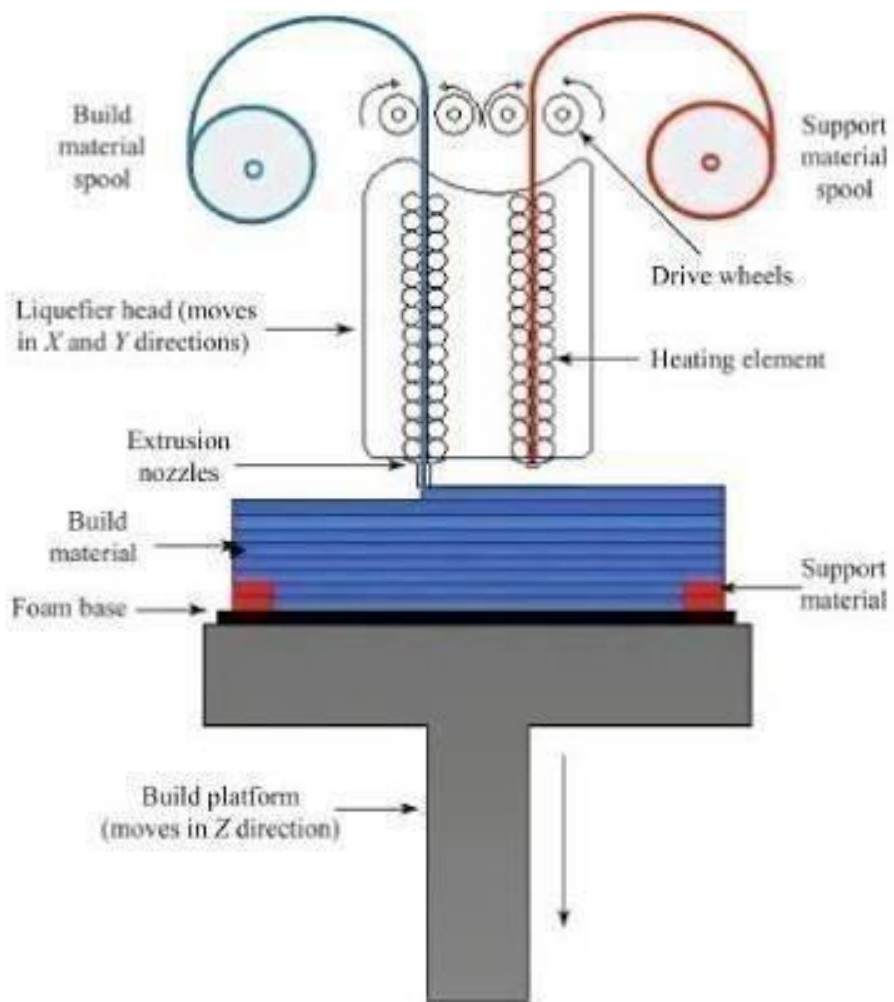

Figure. 2. FDM working process

\subsection{Model Design}

The Catia model in figure 3 is a simple cube design which is converted after in STL format for printing the object in 3D and to measure the Parameters.

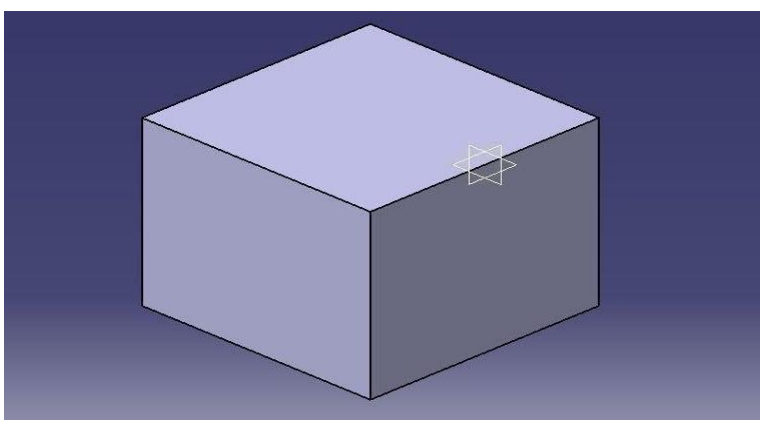

Figure 3. 3D Model design of Cube 


\title{
International Advanced Research Journal in Science, Engineering and Technology
}

\author{
Vol. 6, Issue 3, March 2019
}

\section{CONCLUSION}

Analysis of parameters should be carried out to optimize the 3D printing process. The L9 Orthogonal Array is used to find out the most effective experimental running process for optimization of 3D printing process. From the nine 3D printed cubes, $3^{\text {rd }}$ cube with parameters of Layer thickness (0.3), Layer speed (70) and Infill Density (30) is the most precised cube measured on $\mathrm{cmm}$ machine. The dimensional accuracy should be increased by increasing the layer thickness and layer speed and decreasing the infill density. The optimization of 3D printing process is improved by using this process.

\section{REFERENCES}

[1]. Samir Kumar PANDA, Saumyakant PADHEE, Anoop Kumar SOOD,S.S.MAHAPATRA "Optimization of FDM process parameters using Bacterial Foraging Techniques", 2009,1,89- 97.

[2]. O.Y.Venkatasubbareddy, Palaiam siddikali, S. Mahammad salim "Improving the diamensional accuracy and surface roughness of FDM parts using optimization techniques",2010

[3]. Farzad Rayegani, Godfrey.C, Onwubolu , "FDM process parameter prediction and optimization using group method for data handling (GMDH) and differential evolution(DE)", 25 April 2014

[4]. Raju .B.S. "Establishment of process model for rapid prototyping technique. To enhance the part quality by Taguchi method", $2014,380-389$

[5]. Nilesh kumar, Hemant kumar, Jagdeep Khurmi "Experimental investigation of process model for rapid prototyping technique to enhance the part quality by Taguchi Method”, 2016, 352-360 